\title{
SPECTROSCOPY OF COOL STARS FROM IUE DATA
}

\author{
P. G. Judge \\ Department of Theoretical Physics, \\ University of Oxford, \\ 1 Keble Road, \\ Oxford OX1 3NP, \\ England.
}

\begin{abstract}
.
After nine years of operation the IUE satellite continues to provide valuable spectra of cool stars from 1200 to $3100 \dot{\mathrm{A}}$. The impact of these spectra has been greatest in studies of the outer regions of the atmospheres, above the photospheres, allowing the general properties of stellar chromospheres, transition regions and winds to be established. After outlining these properties, I focus on studies based on high signal-to-noise echelle spectra $\left(\lambda / \Delta \lambda \sim 1.2 \times 10^{4}\right)$ of single stars, showing how high quality emission line profiles have been used to derive constraints on the outer atmospheric structure, which in turn have been used to examine models of heating and mass loss.
\end{abstract}

\section{INTRODUCTION}

Prior to the launch of the International Ultraviolet Explorer (IUE) satellite in 1978, only a few observations of cool stars had been made in the ultraviolet (UV) region below $3000 \dot{A}$ (see e.g. the review by Linsky, 1980). Since then, IUE has revolutionized our knowledge of the outer atmospheres of cool stars, largely through the study of spectral lines, and now we possess a quite detailed knowledge of stellar chromospheres, transition regions (TR's), coronae and winds in the cool half of the HR diagram.

The aim of the present review is to highlight advances in this field using IUE data with high signal-to-noise $(\mathrm{S} / \mathrm{N})$ ratios. Despite the relatively low quality of $I U E$ data compared with modern ground-based spectroscopy (the maximum count/pixel with IUE is 255), data are of sufficient quality in the essentially unexplored and important UV spectral region that great strides have been made in studies of cool stars and other fields of astronomy (see the remarkable book edited by Kondo et al. (1987)).

\section{AN OVERVIEW OF COOL STAR SPECTRA IN THE ULTRAVIOLET}

I give here a brief description of the general characteristics of the spectra of cool stars across the HR diagram. More comprehensive discussions can be found in the reviews of Jordan \& Linsky (1987) and Dupree (1986). Figure 1 (from Dupree, 1986) shows spectra of three stars representing the different basic types of outer atmospheres 
identified in part from IUE observations.

(i) "coronal" stars include all observed dwarfs cooler than $\sim$ FO and lower gravity stars as late as KO III. The UV spectra appear similar to those of regions of various levels of activity in the Sun showing both chromospheric and TR emission lines (up to temperatures of $T_{e} \sim 2 \times 10^{5} \mathrm{~K}$ from $\mathrm{N} V$ 1240). $\mathrm{X}$-ray observations with the EINSTEIN satellite (Ayres et al., 1981) revealed the presence of coronal emission at the levels expected when scaled from a Solar-like outer atmosphere.

(ii) "non-coronal" stars are cooler and more luminous than spectral type K0 III. IUE spectra revealed relatively strong chromospheric emission lines (especially of neutral species), an absence of detectable plasma above $\mathrm{T}_{e} \sim 2 \times 10^{4} \mathrm{~K}$, and asymmetric profiles with enhanced red wings indicating significant velocity gradients, probably associated with a massive wind, in optically thick chromospheric lines (e.g. Mg II $k$ ). No $\mathrm{X}$-rays have yet been detected from single non-coronal stars: upper limits of surface fluxes are much lower than in "coronal" stars.

(iii) "hybrid" stars are mostly luminosity class II stars which lie in the "non-coronal" region of the HR diagram. IUE spectra show evidence both for $10^{5} \mathrm{~K}$ plasma and massive winds of higher terminal velocity than their non-coronal counterparts.

The regions in the HR diagram where the different atmospheric types are found are shown in Figure 2 (from Mullan \& Stencel, 1982).

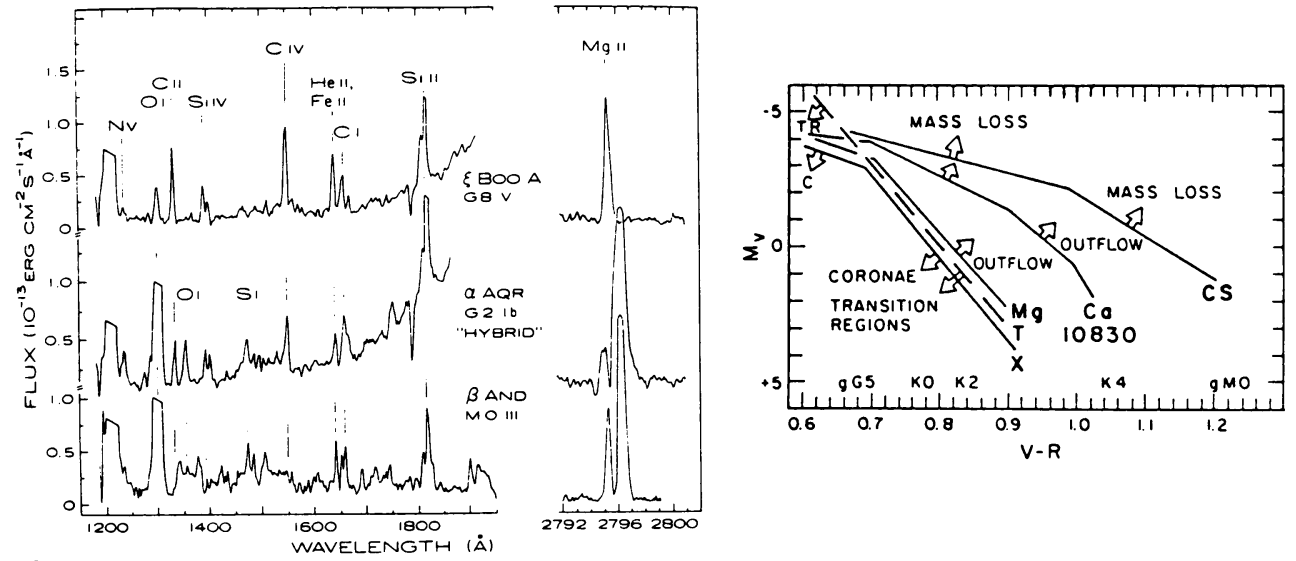

Figure 1: Spectra of 3 cool stars illustrating the characteristic features of "coronal", "hybrid" and "non-coronal" stars. (From Dupree 1986).

Figure 2: An HR diagram showing the regions where different types of outer atmospheric structure are found according to various spectral signatures. "Coronal" stars lie to the left of the lines markes "T" and "X" (from Mullan \& Stencel, 1982).

\section{EXAMPLES OF "HIGH" S/N STUDIES OF THE OUTER ATMOSPHERES OF COOL STARS.}

\section{1 "Coronal" Stars}

Although we can study the Sun's outer atmosphere in much more detail than any other coronal star, spectra of large numbers of such stars have been obtained with IUE which have established important correlations of e.g. "activity" with fundamental 
stellar parameters such as rotation and age which are crucial to the understanding of the mechanisms responsible for heating chromospheres and coronae (e.g. Jordan \& Linsky, 1987). In addition, detailed studies of high-S/N spectra of the brightest individual stars have, by various semi-empirical techniques applied earlier to the Sun, yielded models for the upper chromospheres and TRs of stars of widely differing gravities. Jordan et al. (1987), for example, applied the technique of emission measure analysis (based on the integrated fluxes of emission lines) with additional constraints from observed line widths and ratios to derive models for $5 \mathrm{G}-\mathrm{K}$ dwarf stars. These models span conditions of different regions found in the Sun, and Jordan et al. concluded that the processes determining the structure of the outer atmosphere are basically the same as for the Sun, with the same associated problems (Jordan, 1980).

Lower-gravity "coronal" stars have also been studied using similar techniques (e.g. Brown et al., 1984) and using model- atmosphere approaches (e.g. Eriksson et al., 1983). Similar results to the solar case are found but the lower gravities lead to lower pressures, as expected, and larger scale heights. An important discovery was made by Ayres et al. (1983): in high quality spectra of the primary of Capella (G6 III), lines formed at TR temperatures ( $>5 \times 10^{4} \mathrm{~K}$ ) are significantly red-shifted (by up to $20 \mathrm{~km} \mathrm{~s}^{-1}$ ) relative to the photosphere. Ayres et al. also found similar shifts between TR and chromospheric lines in the 4 other coronal stars examined, and concluded that the shifts probably arise from definite downflows analogous to those observed in the solar network boundaries. Such large-scale flows must be accounted for in the energy and momentum requirements of the TR/ chromosphere.

\section{2 "Non-Coronal" Stars}

Owing to significant differences between UV spectra of "coronal" and "non-coronal" stars, modelling of "non-coronal" atmospheres is at a more basic stage, since solar techniques mentioned above cannot be directly applied. Following early work on rocket spectra by Haisch et al (1977) prior to IUE, advances were initially made in line identifications and excitation mechanisms (see the discussion by Jordan \& Judge, 1984). This work was done in the light of chromospheric density estimates from a valuable diagnostic (line ratios within the C II] $\lambda 2325$ multiplet) by Stencel et al. (1981), which (when modified by up-dated atomic data of Lennon et al., 1985) showed that electron densities are typically $\sim 10^{9} \mathrm{~cm}^{-3}$ for late $\mathrm{K}$ giants, substantially smaller than in the solar chromosphere where $\mathrm{N}_{\mathrm{e}} \sim 10^{11} \mathrm{~cm}^{-3}$ (Vernazza, Avrett \& Loeser, 1981). Figure 3 shows the C II] lines observed in $\alpha$ Tau (K5 III) and $\beta$ Gru (M5 III). Such low chromospheric densities allow radiative processes, such as fluorescence, line-locking, photoionization and line broadening due to multiple scattering to become crucial in forming the observed spectra (Jordan \& Judge, 1984; Judge, 1986a). Nevertheless, detailed empirical studies based on emission measures, linewidth measurements, opacity sensitive line ratios and density sensitive lines have been succesfully applied to three stars ( $\alpha$ Boo (K2 III), $\alpha$ Tau (K5 III) and $\beta$ Gru (M5 III)) to derive useful constraints on the chromospheric emitting regions (Judge, 1986a,b).

Detailed profile modelling of even well-understood lines (e.g. $\mathrm{Mg}$ II $\mathrm{k}$ ) under the conditions derived by Judge $(1986 \mathrm{a}, \mathrm{b})$ is complicated by partial redistribution effects, Doppler diffusion (Basri, 1980) and geometry in spherically expanding atmospheres (Drake \& Linsky, 1983; Drake, 1985). Drake (1985) has computed $\mathrm{Mg}$ II $k$ line profiles, taken these problems into account, and has derived models for the expanding chromosphere of $\alpha$ Boo (Figure 4 ) which also satisfy radio ( $f-f$ ) constraints from the VLA, yielding the first realistic mass loss rate for a typical (single) non-coronal giant. 
Profile modelling work is currently in progress (Judge, Avrett \& Loeser, 1988) using all available data for $\alpha$ Boo in an attempt to unify the various techniques and diagnostics available.
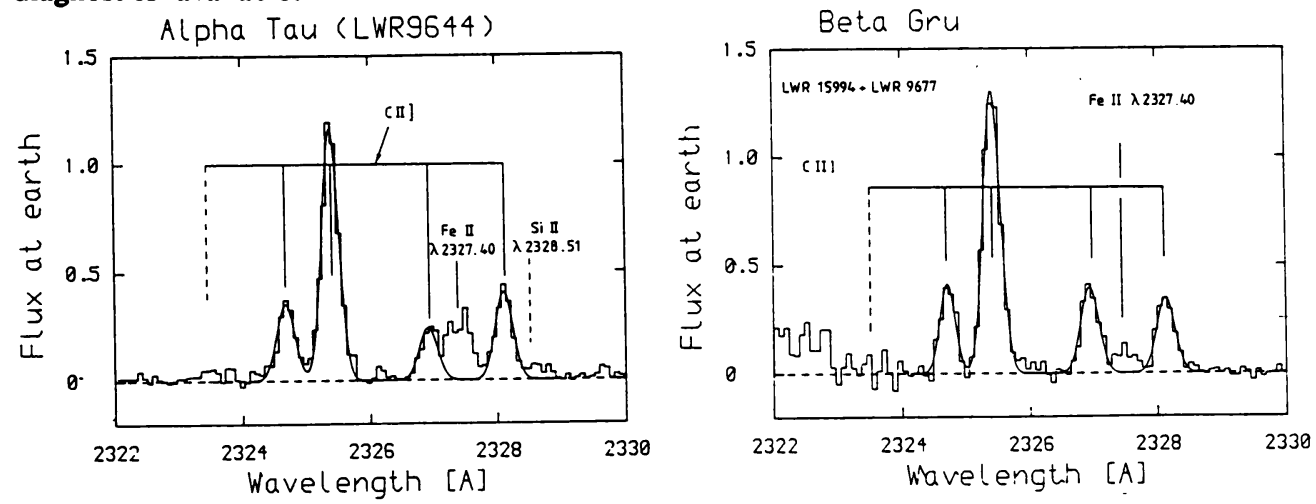

Figure 3: Example of line profiles from IUE echelle spectra. The figure shows observed lines of the density-sensitive C II] multiplet (histograms) and least-square Gaussian fits (solid lines). The fits yield a mean electron densities of $10^{9}$ and $5 \times 10^{8}$ $\mathrm{cm}^{-3}$ for the emitting regions of $\alpha$ Tau and $\beta$ Gru, respectively (From Judge, 1986b).
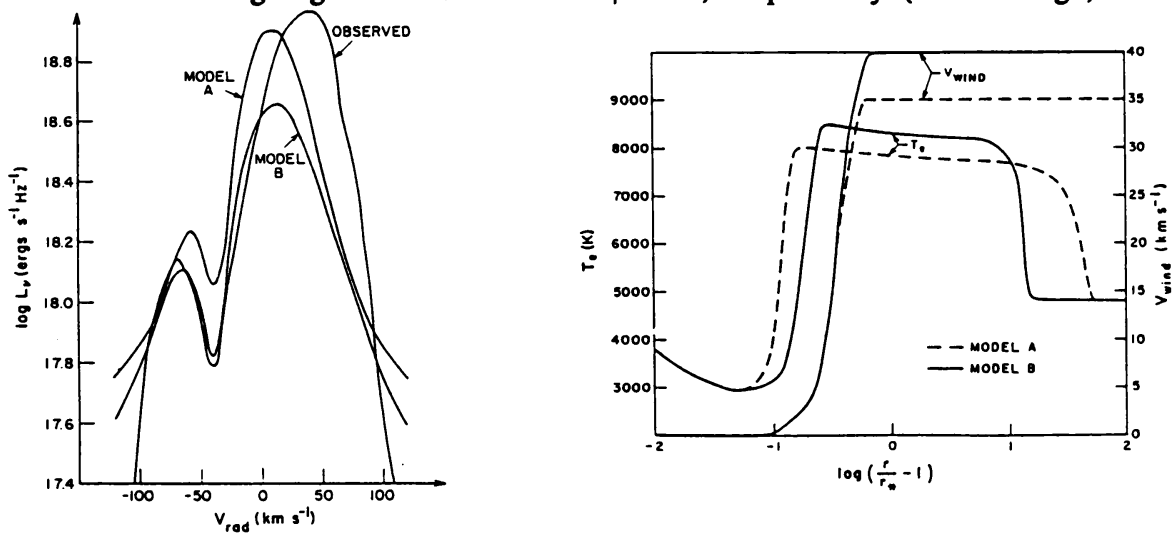

Figure 4: Observed and computed $\mathrm{Mg}$ II $k$ line profiles and wind models from Drake (1985). A mass loss rate of $2 \times 10^{-10} \mathrm{M}_{\odot} \mathrm{yr}^{-1}$ was derived from these profiles.

Perhaps the best constraints from IUE on the outer atmospheres of non-coronal stars have been obtained by studying absorption lines observed when the chromosphere and wind of the primary component of e.g. a 5 Aur system ( $\mathrm{K}$ - supergiant) eclipses the secondary ( a B dwarf). As reviewed by Reimers (1987) radially-dependent chromospheric and wind parameters for the primary have been derived yielding accurate mass loss rates (factor $\sim 3$ uncertainties). This method is, however, restricted to a small region of the HR diagram containing suitable systems. Nevertheless valuable constraints on theoretical models have been derived using these techniques.

Comparisons of theoretical and observed line profiles have been made only for the case of an Alfven wave driven wind model of $\alpha$ Ori (M2 Iab) (Hartmann \& A:rett, 1984). Although able to account for global properties of the spectra (e.g. the radio ( $f-f)$ emission), the model's velocity field differs from that inferred from the 
IUE profiles. Work is currently in progress for acoustic- shock driven wind models (Cuntz et al., 1987), another mechanism currently in favour proposed to account for "non-coronal" chromospheres and winds.

\section{3 "Hybrid" Stars}

Since the (dozen or so) known hybrids have spectral features common to both "coronal" and "non-coronal" stars, techniques applied to these have been extended to the hybrids (e.g. Hartmann et al., 1985). Hartmann et al. found that the UV line fluxes are profiles in $\alpha \operatorname{TrA}$ (K4 II) could be accounted for in a hydrostatic wave-supported atmosphere which could perhaps extend into an Alfven-wave driven wind. However, Mendoza (1984) finds that the wind models which give the best fit to line fluxes predict differential expansion velocities which are too large at $T_{e} \leqslant 6 \times 10^{4}$ $\mathrm{K}$. Also, $\mathbf{S} / \mathrm{N}$ ratios are lower for the visually fainter hybrids than for the brightest "coronal" or "non-coronal" stars and higher $S / N$ observations are necessary for further progress to be made (see below).

\section{FUTURE UV SPECTROSCOPY OF COOL STARS}

IUE has provided the first insight into the outer atmospheric structure of many stars in the cool half of the HR diagram. The IUE spectra indicate that a similar leap in our understanding will occur when the HRS on Hubble Space Telescope (HST) is in operation. Some important improvements over IUE will be: (i) the resolution of previously unresolved lines formed in the chromospheres, winds and TRs of cool stars (see e.g. the partially resolved profiles in Fig. 3). This will enable much deeper comparisons of theory and observation to be made concerning the heating and momentum deposition in cool star outer atmospheres; (ii) the higher $S / N$ will reveal much higher quality emission line profiles for lines already observed with IUE. It will allow the detection of weaker absorption features (e.g. wind components) and emission components (e.g. density- sensitive quadrupole lines of C III], faint emission components in the wind), if present, and the ability to study variations at the level of a few percent.

\section{REFERENCES.}

Ayres T.R., Linsky J.L., Vaiana G.S., Golub L. \& Rosner R.,1981. Astrophys. J. $250,293$.

Ayres T.R., Stencel R.E., Linsky J.L., Simon T., Jordan C., Brown A. \& Engvold O., 1983. Astrophys. J. 274, 801.

Basri G.S., 1980. Astrophys. J. 242, 1133.

Brown A., Jordan C., Stencel R.E., Linsky J.L. \& Ayres T.R., 1984. Astrophys. J. 283, 731 .

Cuntz M., Hartmann L. \& Ulmschneider P., 1987. In "Circumstellar Matter", IAU Symp. 122, 325. (Eds. Appenzeller I. and Jordan C.). Reidel (Dordrecht).

Drake S.A. \& Linsky J.L., 1983. Astrophys. J. 273, 299.

Drake S.A., 1985. In 'Progress in Stellar Spectral Line Formation Theory',(Eds. Beckman \& Crivellari), p.351. Reidel (Dordrecht).

Dupree A.K., 1986. Ann.Rev. Astron. Astrophys. 24, 377.

Eriksson K., Linsky J.L, \& Simon T., 1983. Astrophys. J. 272, 665.

Haisch B.M., Linsky J.L., Weinstein A. \& Shine R.A., (1977). Astrophys. J. 214, 785. 
Hartmann L., Jordan C., Brown A. \& Dupree A.K., 1985. Astrophys. J. 296, 576. Hartmann L. \& Avrett E.H., 1984. Astrophys. J. 284, 238.

Jordan C., 1980. Astron. Astrophys. 86, 355.

Jordan C., Ayres T.R., Brown A., Linsky J.L. \& Simon T., 1987. Mon. Not. R. astr. Soc. 225, 903.

Jordan C. \& Judge P.G., 1984. Physica Scripta T8, 43.

Jordan C. \& Linsky J.L., 1987. In "Exploring the Universe with the IUE Satellite", p. 259. Eds. Kondo et al., Reidel (Dordrecht).

Judge P.G., 1986a. Mon. Not. R. astr. Soc. 221, 119.

Judge P.G., 1986b. Mon. Not. R. astr. Soc. 223, 239.

Kondo Y. et al., (eds.), 1987. "Exploring the Universe with the IUE Satellite", Reidel (Dordrecht).

Lennon D.J., Dufton P.L., Hibbert A. \& Kingston A.E., 1985. Astrophys. J. 294, 200.

Linsky, J.L., 1980. Ann. Rev. Astron. Astrophys. 18, 439.

Mendoza B.M., 1984. D. Phil. Thesis, University of Oxford.

Mullan D.J. \& Stencel R.E., 1982. In "Advances in UV Astronomy: Four Years of IUE Research", NASA CP-2238, p. 235.

Reimers D., 1987. In "Circumstellar Matter", IAU Symp. 122, 307. (Eds. Appenzeller I. and Jordan C.). Reidel (Dordrecht).

Stencel R.E., Linsky J.L, Brown A., Jordan C., Carpenter K.G., Wing R.F. \& Czyzak S., 1981. Mon. Not. R. astr. Soc. 196, 47P.

Vernazza J.E., Avrett E.H. \& Loeser R., 1981. Astrophys. J. Suppl. 45, 635.

\section{DISCUSSION}

CRIVELIARI It is well known that the Mg II $h$ and $k$ interstellar component is strong enough (even within a few parsecs from the sun) to significantly alter the profile in the emission cores. We have evidence that (Vladilo et al., 1987, in press : Astron. Astrophys.) in specific cases the local interstellar medium (LISM) contamination (the precise wavelength of the IISM component is derived by Crutcher's LISM flow velocity) can reverse the expected $V / R$ asymmetry of the emission core.

JUDGE I agree that the LISM is an important factor which should be taken into account when examining Mg II profiles. Space Telescope should, with its factor 10 gain in resolution over IUE, enable the "wind" and IISM components to be separated better.

EBBEIS You mentioned several times your anticipation that observations to be made with the space Telescope High Resolution spectrograph will make important new contributions to your field. Could you identify what you consider to be the most important frRs performance parameters for your work, and what types and precisions of calibrations will be required.

JUDGE The most important single improvement over IUE will be the increased resolution $(\simeq 3 \mathrm{~km} / \mathrm{s})$ which will allow, for the first time, the profiles of optically thin/thick lines to be examined, yielding tight constraints on energy and momentum deposition. Accurate ( $<0.5$ kws) radial velocities could be very useful. Absolute photometric calibration better than $\times 108$ would be adequate, but it would be nice to measure variability in line fluxes at a level $\simeq 18$. 